\title{
Splitting of Cell Clusters and Bifurcation of Bryozoan Branches
}

\author{
Lloyd Goldwasser, $\dagger$ P. K. Maini $¥$ and J. D. Murray \\ Centre for Mathematical Biology, Mathematical Institute, 24-29 St Giles, \\ Oxford $O X 13 L B, U . K$.
}

(Received and accepted 24 May 1988)

\begin{abstract}
We present a cell mechanical model that exhibits the pattern-forming behaviour of a cluster of cells at the growing tips of bryozoan branches. The crucial event in the production of the overall branching pattern is the splitting of the cluster into two clusters, rather than the formation of the cluster from a uniform distribution. In simulations, the uniform cell distribution initially evolved, as indicated by a linear analysis, either to a spatially patterned state, or to a temporally oscillating state. We suggest that the same processes responsible for the formation and behaviour of the cell cluster may aiso be responsible for tip branching.
\end{abstract}

\section{Introduction}

Branching structures such as trees, corals and bryozoans are created by iteration of two separate processes: the growth of branches and the splitting of one branch into two. The characteristic and often striking differences in branching structure among taxa result from differences in the timing and location of the splitting events. Those differences can be modelled phenomenologically by constructing rules for growth and branching, and then describing their resulting patterns (for example, Bell, 1976; Fisher \& Honda, 1979; Gardiner \& Taylor, 1982; McKinney \& Raup, 1982; Niklas, 1982; cf. Waller \& Steingraeber, 1985; Bell, 1986; Goldwasser, 1988). Such models, however, do not answer, or even pose, questions about the actual processes that underlie and determine the rules. Other models, such as those of reaction diffusion systems (for example, Lacalli \& Harrison, 1987; Harrison \& Kolar, 1988), depend on as yet undiscovered morphogens to establish the branching patterns. In this paper we present a mechanical model, based on cellular processes known to be involved in both branch growth and splitting in arborescent bryozoans, and show that it exhibits both growth and splitting.

Many models of cellular mechanics (for example, Oster et al., 1983; Murray \& Oster, $1984 a, b)$ investigate the initial formation of pattern as deviations from initially uniform conditions; in such cases a linear stability analysis about the uniform steady states provides a reasonably good prediction of the ultimate spatially heterogeneous pattern formed. For bryozoan growth, however, the pattern-forming event is the splitting of one cluster of cells into two (Schneider, 1963; Schneider \& Kaissling, 1964); and a stability analysis of a non-uniform distribution is rarely possible or

$\dagger$ Present address: Department of Biological Sciences, Stanford University, Stanford, California 94305, U.S.A.

$\ddagger$ Present address: Deparment of Mathematics, University of Utah, Salt Lake City, Utah 84112, U.S.A. 
feasible. Here we have used simulations of cell movements to investigate the events after the cluster of cells is formed. These simulations display not only the formation of the initial pattern predicted by the model, but also the subsequent splitting of one cluster into two.

\section{Branching in Bryozoans}

Growth in bryozoans is distal, with new units (zooids) added to the end of branches regardless of whether splitting occurs (see Fig. 1). In one of the few studies of the growing tips of bryozoans, Schneider (1963) reported a group of cells in Bugula with striking behaviour:

"While there is limited random movement of the individual cells, the degree of cell co-ordination is remarkable. All these cells are somehow oriented to the centre of the cell plate, which is rhythmically expanding or contracting, or even twisting."

He concludes, "How this locomotive activity and co-ordination is brought about is quite unknown." Our model specifically addresses these questions.

We focus on the behaviour of these cells because of their effect on both the growth and splitting of the branches (Schneider, 1963; Kaissling, 1963; Lutaud, 1961, Tavener-Smith \& Williams, 1972). The cells lie on the inner, domed surface of a growing tip, where they secrete the cuticle as the branch grows (Tavener-Smith \& Williams, 1972). The new cuticle is inserted directly into the existing cuticle, as illustrated in Fig. 2. Older cuticle at the periphery of the dome becomes calcified

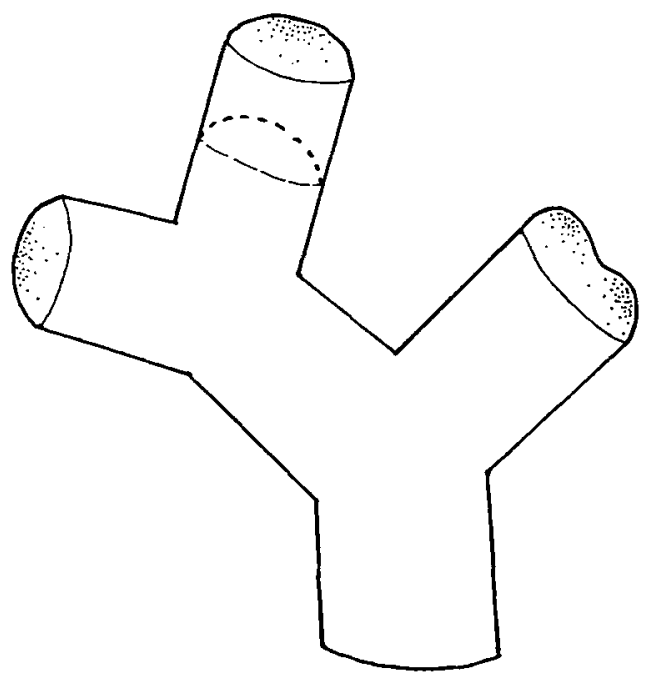

FIG. 1. Diagram of an arborescent bryozoan colony, such as Bugula sp., showing the cells at the growing tips. The cluster of cells on the rightmost branch has split, and that branch is beginning to form two daughter branches. For simplicity the branches are depicted as continua rather than as divided into discrete zooids (which occurs only proximally from the tip). 


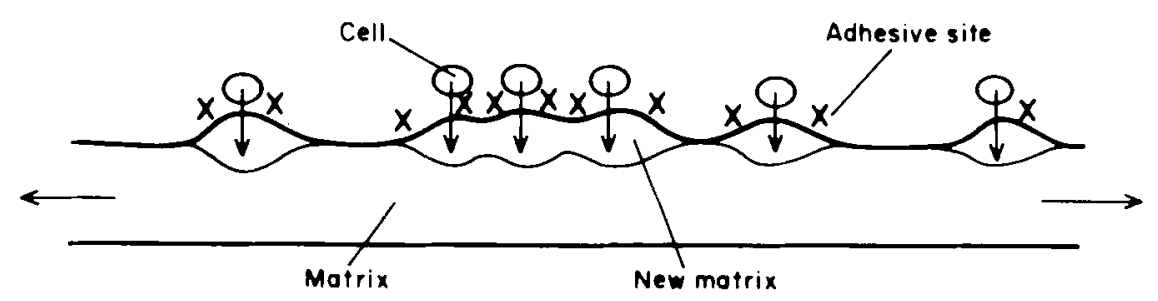

F1G. 2. Diagrammatic cross section through the cuticle at the tip of a bryozoan branch, laid flat. The cells sit on the inside surface of the tip and secrete cuticle. The new cuticle is inserted directly into the existing cuticle, which spreads out, carrying with it the cells and adhesive sites.

and, after being pushed outward and becoming part of the cylinder of the branch, falls behind the forward-growing tip. The direction of branch elongation depends on the location of the cells within the tip. Splitting of the cluster into two coincides with the splitting of one branch into two daughter branches (Schneider, 1963).

A few other details are known about these cells. They do not themselves reproduce; new cells are recruited proximally from within the branch (Schneider, 1963; TavenerSmith \& Williams, 1972). The motion of individual cells is rapid on the time scale of branch growth (personal observation).

We take the splitting of the cluster of cells to be the initial event in branching, and do not attempt to model the subsequent alterations in the shape of the dome (Fig. 1) or the establishment of the two independent daughter branches.

\section{Model for Cell Patterning at Tips of Bryozoan Branches}

For mathematical simplicity, we construct a model on a one-dimensional domain to focus on the behaviour of the clusters of cells rather than on the more complex dome-like structure of the branch tips. We use $r(x, t)$ for the density of the matrix, or cuticle; $a(x, t)$ for the density of adhesive sites; and $n(x, t)$ for the density of the cells at each point $x$ at time $t$. The cells and the matrix are both observable, while the adhesive sites are postulated. We introduce the adhesive sites because some attractive entity is necessary for the production of patterns. In itself, convection by the spreading matrix can only tend to break up patterns as it carries cells out of the domain.

Our model is based essentially on two processes: the spreading of the matrix with the convection of cells and adhesive sites with it, and the secretion of matrix and adhesive sites by the cells. The cells thus follow a gradient in adhesive sites-of their own creation-as they are carried by the matrix. This scheme is illustrated in Fig. 2. The movement of cells up such a gradient, known as haptotaxis, is a general and widely observed phenomenon in cell behaviour (see, for example, Harris, 1983).

The model equations are:

$$
r_{\mathrm{t}}=\underset{\text { secretion }}{\zeta n}-\underset{\text { calcification }}{\lambda r}+c\left(r r_{x}\right)_{x}
$$




$$
\begin{aligned}
& a_{i}=\underset{\text { secretion }}{b n}-\underset{\text { decay }}{\mu a}+c\left(a r_{x}\right)_{x} \\
& n_{t}=\underset{\text { convection }}{d n_{x x}}-\underset{\text { diffusion }}{h\left(n a_{x}\right)_{x}}+c\left(n r_{x}\right)_{x} \\
& \text { haptotaxis convection }
\end{aligned}
$$

where subscripts denote partial differentiation. The parameter $c$ represents the rate at which the matrix spreads out as new matrix is inserted, and, in the similar terms in the second and third equations, the rate at which the adhesive sites and cells are carried with it. The rate of secretion of matrix per cell is $\zeta$; the loss of matrix by calcification is $\lambda$ per unit of matrix. Similarly, in eqn (1b), $b$ represents the rate of secretion of adhesive sites per cell, and the decay of adhesive sites occurs at the rate $\mu$. In eqn (1c), $d$ is the diffusion constant and $h$ is the rate at which cells move up a gradient in adhesive sites. Because the cells are motile, unlike the matrix and adhesive sites, we include a diffusion term only for the cells. There is no term for cell recruitment because these cells are non-mitotic (Schneider, 1963; Tavener-Smith \& Williams, 1972). We have chosen linear secretion and calcification or decay terms for their simplicity; it is not necessary to invoke more complicated behaviour to produce the patterns observed.

\section{Linear Analysis for Pattern Initiation}

We denote the mean cell density throughout the domain by $N$, the original length of the domain by $L_{0}$, and its current length by $L$. We make the following nondimensional substitutions:

$$
\begin{array}{cccc}
n^{*}=n / N, & a^{*}=a h /\left(\mu L_{0}^{2}\right), & r^{*}=r c /\left(\mu L_{0}^{2}\right), & x^{*}=x / L, \\
t^{*}=t \mu L_{0}^{2} / L^{2}, & \gamma=L^{2} / L_{0}^{2}, & \zeta^{*}=c N \zeta /\left(\mu^{2} L_{0}^{2}\right), & \lambda^{*}=\lambda / \mu, \\
b^{*}=b h N / \mu^{2} L_{0}^{2}, & d^{*}=d /\left(\mu L_{0}^{2}\right) .
\end{array}
$$

The resulting non-dimensional system becomes, on dropping the asterisks for notational convenience,

$$
\begin{aligned}
& r_{t}=\gamma(\zeta n-\lambda r)+\left(r r_{x}\right)_{x} \\
& a_{t}=\gamma(b n-a)+\left(a r_{x}\right)_{x} \\
& n_{t}=d n_{x x}-\left(n a_{x}\right)_{x}+\left(n r_{x}\right)_{x} .
\end{aligned}
$$

If $n$ is constant over the domain, then the non-dimensionalization eqn (2) implies that $n=1$. The uniform steady states for $(r, a, n)$ are $(0,0,0)$ and $(\zeta / \lambda, b, 1)$; the latter is the biologically relevant one. We linearize about this steady state and look for solutions of the form:

$$
\left[\begin{array}{c}
r-\zeta / \lambda \\
a-b \\
n-1
\end{array}\right] \propto \exp [\sigma t+i k x]
$$

where $k$ is the wave number of the perturbation (which is inversely proportional to the wavelength of the perturbation), and $\sigma$ the rate of growth of perturbation with wave number $k$. When $\operatorname{Re}(\sigma)$ is positive the perturbation grows; when $\operatorname{Re}(\sigma)$ is 
negative it decreases. Substitution of this form into the linear equations gives the following dispersion relation between $k$ and $\sigma$ :

$$
\begin{gathered}
\sigma^{3}+\left[(d+\zeta / \lambda) k^{2}+\gamma(\lambda+1)\right] \sigma^{2}+\left[d \zeta k^{4} / \lambda+k^{2} \gamma(\zeta / \lambda+d \lambda+\zeta+d-b)+\gamma^{2} \lambda\right] \sigma \\
+\left[k^{4} \gamma \zeta(d / \lambda+b-b / \lambda)+k^{2} \gamma^{2}(d \lambda+\zeta-b \lambda)\right]=0
\end{gathered}
$$

In the spatially uniform situation, when $k=0$, the dispersion relation reduces to

$$
\sigma^{3}+\gamma(\lambda+1) \sigma^{2}+\gamma^{2} \lambda \sigma=0
$$

which has the solution $\sigma=0$ with the other two roots being negative. Because no roots are positive, the system is stable to spatially homogeneous solutions. When $k \neq 0$, the cubic eqn (4) has either three real roots for $\sigma$ or one real root and two
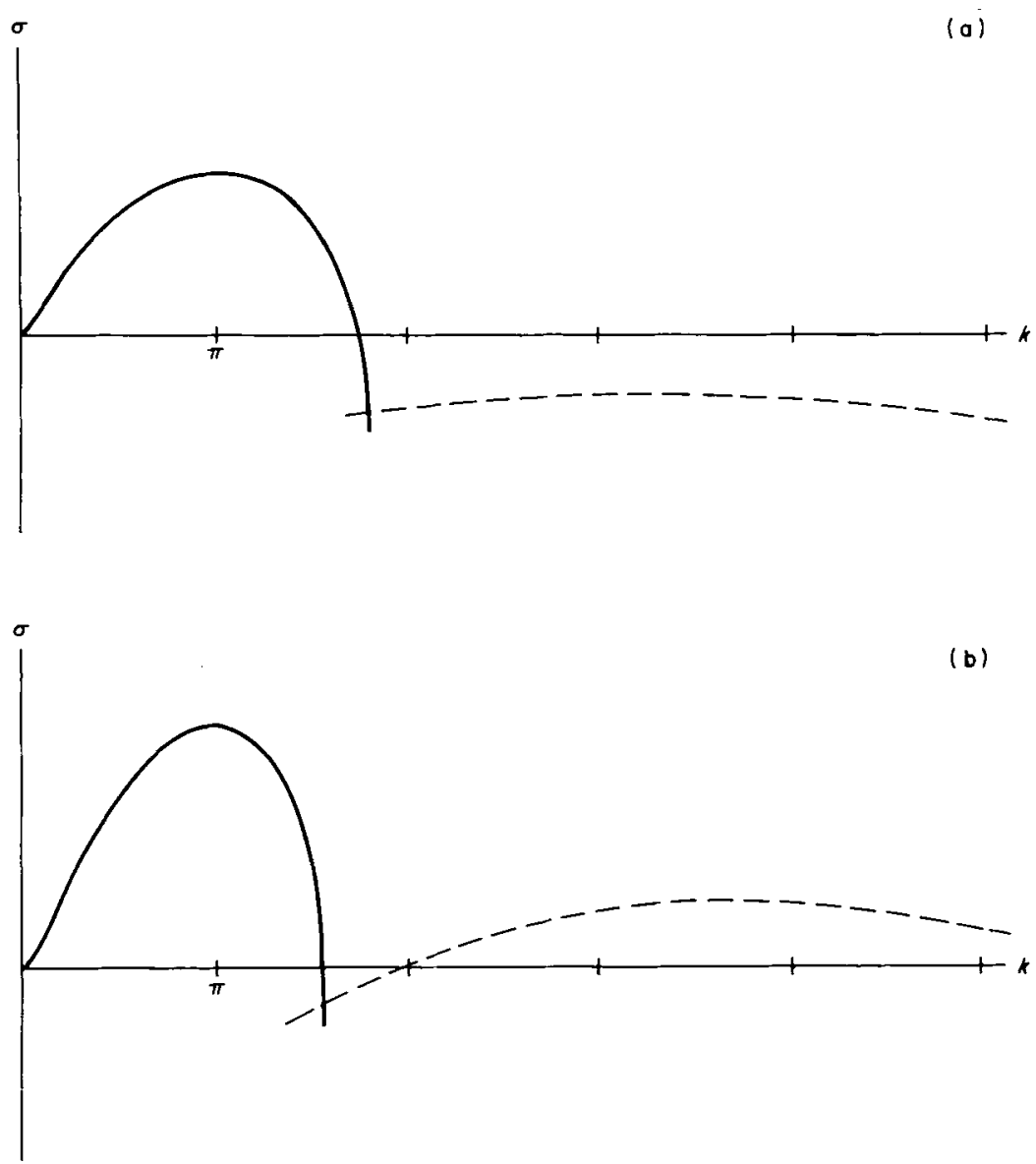

FIG. 3. (a) A typical dispersion relation. Parameter values: $d=10, b=200, \lambda=20, \gamma=12, \zeta=10$. For low values of $k$ the real root $(-)$ is both positive and greater than the real part of the complex roots. For larger $k$, the real part of the complex roots (- _-) is greater than the real root but still negative; these disturbances do not grow. (b) A dispersion relation in which a complex eigenvalue has a real part positive and greater than the real eigenvalue of that wave number. The complex part suggests temporal wave-like oscillations. 
complex conjugate roots. The behaviour of a disturbance with wave number $k$ depends on the sign of the real part of that root of eqn (4) (real or imaginary) whose real part is greatest. When that greatest real part is positive, the disturbance grows. For some values of the parameters, the root with greatest real part is real and positive for a range of $k$, suggesting that the initially uniform cell distribution will evolve to a spatially heterogeneous one. Figure 3(a) shows a dispersion relation in such a case. For some values of the parameters, however, some values of $k$ give complex roots $\sigma$ whose real part is both positive and greater than the real root for that $k$. In such cases, the complex root determines not only whether that mode grows but also that its behaviour is oscillatory (cf. Murray, in press). Figure 3(b) shows a dispersion relation in which there is a range of $k$ for which $\sigma$ is complex and has a positive real part which is greater than the positive real root.

Although we can identify the fastest-growing wave number, $k_{m}$, linear growth is indicated with other wave numbers. From eqn (4) it can be shown that all modes with

$$
0<k<[(b \lambda-d \lambda-\zeta) /(d / \lambda+b-b / \lambda)] \gamma / \zeta
$$

exhibit exponential growth. It is not possible to adjust the parameters, as is typical in linear theory of most pattern formation models (Murray, in press), to isolate one wave number for growth while all others decay. The dispersion relation $\sigma=\sigma(k)$ passes through the origin for all values of the parameters: this tethering to the origin is a direct consequence of the lack of mitosis (Schneider, 1963; Tavener-Smith \& Williams, 1972) among the tip cells. Here, whenever pattern can form, there is a range of many growing wave numbers; the analysis can only single out the wave number that initially grows fastest. In this case the boundary conditions may play a crucial role in determining which pattern develops. Although linear theory provides a predictive guide, numerical simulations are required to produce the spatial patterns that are solutions of the full non-linear system.

\section{Numerical Simulations for Spatial Patterns}

For the simulations we used a simple upwind scheme-a type of Galerkin method (Mitchell \& Griffiths 1980) - that we found to be stable for a wide range of parameter values. Considerable care is required in setting the boundary conditions to reflect the developmental process. Although zero-flux conditions were appropriate for the cells, which remain within the domain of the tip, the matrix continually spreads out of the domain, carrying adhesive sites with it; it was both biologically and mathematically necessary for the flux of matrix and adhesive sites to be non-zero.

Starting with small random perturbations about the non-trivial steady state, the simulations produced the patterns predicted by the linear analysis: either one cell cluster, when $k_{m}=\pi$, or two clusters when $k_{m}=2 \pi$. Although such results are common in studies of pattern formation, they are less informative here. The significant occurrence here is not the initial formation of a cluster-which exists for most of the duration of a branch-but the splitting of that cluster into two clusters that subsequently diverge as the daughter branches grow out. Changes in a pattern after its initial growth from uniform conditions can be due only to non-linearities 
(a)
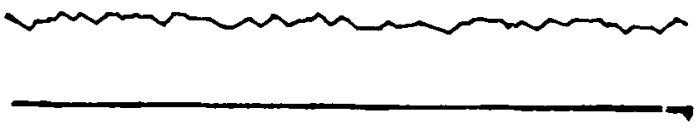

(b)

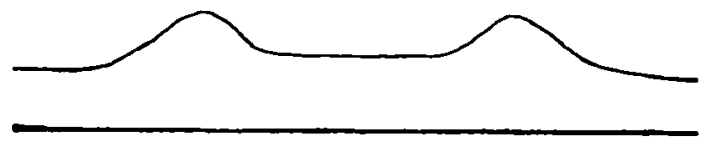

(c)

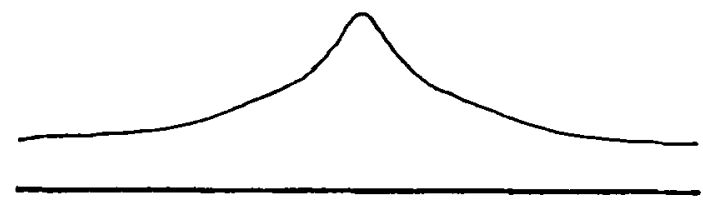

(d)

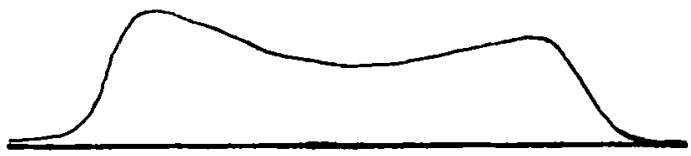

Fig. 4. Simulation showing the formation, from initial conditions (a), of two clusters of cells (b), their merging (c), and their subsequent splitting (d).

in the model; here, the non-linear convective matrix term alters the stability of the cluster of cells, making splitting possible.

The interesting and important feature of the model is that it produced splitting of a formed cluster of cells. Unexpectedly, splitting occurred not only when $k_{m}=2 \pi$ (two clusters form initially), but also when $k_{m}=\pi$ (one cluster forms initially). Thus a single set of parameter values can produce a single cluster arising from random perturbations about uniform initial conditions and its subsequent splitting into two clusters.

The presence of growing modes with complex eigenvalues indicates the possibility of oscillations or travelling waves. Indeed, in some simulations, two initial clusters merged into a single cluster and then split into two again (Fig. 4). The model and simulations can thus capture much that is known about the movements of these cells.

\section{Discussion}

Most models of pattern formation are concerned with the production of pattern from initially uniform conditions. This model exhibits such capabilities as well as the more complex emergence of new patterns from an already patterned state. 
Our model suggests that the mechanism involved in the splitting of bryozoan branches is the same as that involved in normal branch elongation: branching is thus a natural extension of growth. Importantly, splitting may occur without change in parameter values.

Many of the patterns produced were transients rather than steady state solutions. Branching occurs whenever the cluster splits, even if the distribution is not a steady state; the splitting is marked by daughter branches at that point. The transient behaviour of the cells thus affects the subsequent form of a bryozoan colony, and the colony preserves a history of their behaviour.

Oscillatory and wave behaviour have also been observed in the cells at the tips of the encrusting bryozoan Membranipora membranacea (Lutaud, 1961). Although the cells are fixed on the inner surface of the cuticle, waves of cell movement sweep across them from one side of the tip to the other. In this case splitting is associated with the breaking up of one wave into two waves.

The co-ordination of movements of the cells, remarked upon by Schneider (1963), is produced in this model by each cell acting only according to its local conditions; there is no need for long-range transmission of information or a single source of information to guide their movements.

Most of the components of our model are well documented in the literature. We postulate the existence of adhesive sites to provide an aggregating force that counters the convective effects othe spreading matrix. The decay of the adhesive sites also seems plausible and is necessary for pattern formation. A chemo-attractant with similar behaviour would of course produce similar patterns.

The rate of convection may actually depend on the number of cells between each point and the centre of the domain, rather than on the gradient in matrix density. However, we do not pursue this refinement at this stage. Nor does our model address the subsequent events, triggered by the splitting of the cell cluster, that are responsible for the establishment and geometry of the two independent daughter branches.

It is well known that widening the domain affects the formation of pattern (see, for example, Murray, 1981, in press). In encrusting bryozoans the width of the tip affects the probability of branching (Banta \& Holden, 1974), and a gradual widening of branches from one bifurcation to the next has been seen in some Bugula (personal observation). Such changes can be reflected in our model by increasing $\gamma$, which is a measure of scale [see eqn (2)], as a branch grows.

Although the cells at the branch tips do not divide, their number is not constant: recruitment from proximal regions of the branch balances the halving that occurs during branching. Our non-dimensionalized variables $\zeta$ and $b$ increase linearly with $N$, the mean density of cells; and the dispersion relation and fastest growing wavenumber $k_{m}$ change with them. The magnitude and even the direction of the change in $k_{m}$ depends on the particular values of the other parameters. In the model here we have kept $N$ constant because the recruitment of cells is slow relative to the time scale of their motions. In further studies we intend to investigate the patterns formed as $N$ increases during branch growth. It would also be interesting to consider a more realistic dome-shaped tip. The present model, however, seems to capture the principal elements of tip branching. 
L. G. and P. K. M. would like to acknowledge support of the Science and Engineering Research Council of Great Britain through a grant (GR/C/13573) to the Centre for Mathematical Biology, University of Oxford. We also wish to thank Professor Y. Hosono and Drs. V. S. Manoranjan and P. Grindrod for helpful discussions.

\section{REFERENCES}

Banta, W. C. \& Holden, P. M. (1974). Bud size alone does not control bifurcation in Schizoporella unicornis floridana. (Bryozoa, Cheilostomata). Chesapeake Sci. 15, 104-109.

BELL, A. (1976). Computerized vegetative mobility in rhizomatous plants. In: Automata, Languages, Development (Lindemayer, A. \& Rozenberg, G., eds) pp. 3-14. Holland: North-Holland.

BelL, A. D. (1986). The simulation of branching patterns in modular organisms. Phil. Trans. R. Soc. Lond. B313, 143-159.

Fisher, J. B. \& HONDA, H. (1979). Branch geometry and effective leaf area: A study of Terminaliabranching pattern. 1. Theoretical trees. Am. J. Bot. 66, 633-644.

Gardiner, A. R. \& TAYlor, P. D. (1982). Computer modelling of branching growth in the Bryozoan Stomatopora. N. Jb. Geol. Palaont. Abh. 163, 389-416.

GOLDWASSER, L. (1988). Branching patterns, generating rules, and astogenetic trajectories in arborescent bryozoans. J. theor. Biol. 132, 179-201.

HARRIS, A. K. (1983). Cell migration and its directional guidance. In: Cell Interactions and Development: Molecular Mechanisms (K. Yamada, ed.) pp. 123-151. New York: Wiley.

HARRISON, L. G. \& KOLǍ̃, M. (1988). Coupling between reaction-diffusion prepattern and expressed morphogenesis, applied to desmids and dasyclads. J. theor. Biol. 130, 493-515.

Kaissling, K.-E. (1963). Die phototropische Reaktion der Zoide von Bugula avicularia. L. Z. Vergl. Physiol. 46, 541-594.

LACALLI, T. C. \& HARRISON, L. G. (1987). Turing's model and branching tip growth: relation of time and spatial scales in morphogenesis, with application to Micrasterias. Can. J. Botany. 65, 1308-1319.

LUTAUD, G. (1961). Contributions à l'étude du bourgeonnement et de la croissance des colonies chez Membranipora membranacea (Linn) Bryozoaire chilostome. Ann. Soc. r. zool. Belg. 91, 157-300.

MCKINNEY, F. K. \& RAUP, D. M. (1982). A turn in the right direction: simulation of erect spiral growth in the bryozoans Archimedes and Bugula. Paleobiology 8, 101-112.

Mitchell, A. R. \& Griffiths, D. F. (1980). The Finite Difference Method in Partial Differential Equations. Chichester: Wiley.

MURRAY, J. D. (1981). A pre-pattern formation mechanism for animal coat patterns. J. theor. Biol. 88, $161-199$.

Murray, J. D. (1988). Mathematical Biology. Heidelberg: Springer (in press).

Murray, J. D. \& Oster, G. F. (1984a). Generation of biological pattern and form. IMA J. Math. Appl. in Medic. \& Biol. 1, 51-75.

MurRay, J. D. \& OSTER, G. F. (1984b). Cell traction models for generating pattern and form in morphogenesis. J. math. Biol. 19, 265-279.

NIKLAS, K. J. (1982). Computer simulations of early land plant branching morphologies: canalization of patterns during evolution? Paleobiology 8, 196-210.

Oster, G. F., Murray, J. D., \& Harris, A. K. (1983). Mechanical aspects of mesenchymal morphogenesis. J. Embryol. exp. Morph. 78, 83-125.

SCHNEIDER, D. (1963). Normal and phototropic growth reactions in the marine bryozoan Bugula avicularia. In: The Lower Metazoa. (E. C. Dougherty, ed.) pp. 357-371. Berkeley: University of California Press.

SCHNEIDER, D. \& Kaissling, K.-E. (1964). Wachstum und Phototropismus bei Moostieren. Naturwissenschaften 51, 127-134.

TAVENER-SMITH, R. \& WILliams, A. (1972). The secretion and structure of the skeleton of living and fossil bryozoa. Phil. Trans. Royal Soc. Lond. B264, 97-159.

WAller, D. M. \& Steingraeber, D. A. (1985). Branching and modular growth: Theoretical models and empirical patterns. In: Population Biology and Evolution of Clonal Organisms (J. B. C. Jackson, L. W. Buss \& R. E. Cook, eds) pp. 225-257. New Haven: Yale University Press. 